\title{
36
}

\section{Quantification and analysis of three-dimensional shape changes during epithelial morphogenesis.}

Lana Fuhrmann ${ }^{1}$, Joris Paijmans ${ }^{2}$, Carl Modes ${ }^{1,3}$, Frank Juelicher $2,1,4$, Natalie Dye ${ }^{1}$

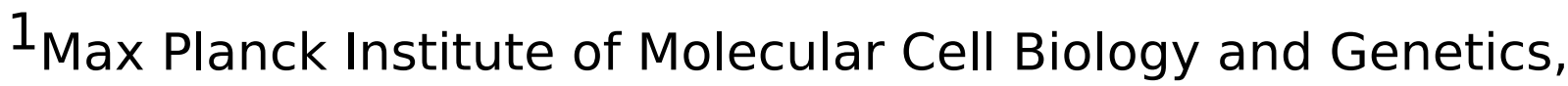
Pfotenhauerstrasse 108, Dresden, Germany. ${ }^{2}$ Max Plank Institute for the Physics of Complex Systems, Nöthnitzer Str. 38, 01187 Dresden, Germany. ${ }^{3}$ Center for Systems Biology Dresden, Pfotenhauerstrasse 108, Dresden, Germany. ${ }^{4}$ Biotechnologisches Zentrum, Technische Universität Dresden, Tatzberg 47/49, Dresden, Germany

\section{Abstract Text}

Changes of 3D tissue architecture during development and morphogenesis are orchestrated through multiple coordinated behaviors. Understanding these processes in a developing tissue requires holistic analysis of cell and tissue shape changes throughout development. Using light sheet and confocal microscopy, we aim to determine how individual cell behaviors collectively transform the shape of epithelial tissues in three dimensions.

A morphogenetic process that is characterized by a complex threedimensional shape change is the eversion of the Drosophila wing disc, by which the folded wing disc transitions to a flat, bi-layered epithelium. Although the molecular cues patterning the wing disc have been investigated thoroughly, little is understood about the changes in epithelial shape and the physical mechanisms underlying wing disc eversion. To systematically investigate these processes, we quantify tissue curvature across different regions of the wing disc, using multi-view light sheet imaging and an array of customized image analysis tools. Contributions of cell shape changes as well as local changes in curvature to global tissue shape are examined based on a 3D representation of the apical cell network. This is based on a segmentation of the cell network generated through a 
projection of the tissue surface over different imaging angles.

In the wing disc we observe changes in tissue curvature followed by an increase in tissue area, while on cellular level we observe a change in the orientation of cell elongations and anisotropic expansion of apical cell areas. Based on these results, we are exploring the hypothesis of apical cell packing geometry being related to tissue curvature and that transitions in tissue shape are mediated by changes in the patterns of cellular packing geometries. Together, this provides an example how light sheet microscopy can be used to thoroughly understand cellular and tissue level contributions of epithelial shape in 3D. 